\title{
Hybrid Erythrocyte Liposomes: Functionalized Red Blood Cell Membranes for Molecule Encapsulation
}

\author{
Sebastian Himbert, Matthew J. Blacker, Alexander Kihm, Quinn Pauli, Adree Khondker, \\ Kevin Yang, Sheilan Sinjari, Mitchell Johnson, Janos Juhasz, Christian Wagner, \\ Harald D. H. Stöver, and Maikel C. Rheinstädter**
}

The modification of erythrocyte membrane properties provides a new tool towards improved drug delivery and biomedical applications. The fabrication of hybrid erythrocyte liposomes is presented by doping red blood cell membranes with synthetic lipid molecules of different classes (PC, PS, PG) and different degrees of saturation (14:0, 16:0-18:1). The respective solubility limits are determined, and material properties of the hybrid liposomes are studied by a combination of X-ray diffraction, epi-fluorescent microscopy, dynamic light scattering (DLS), Zeta potential, UV-vis spectroscopy, and Molecular Dynamics (MD) simulations. Membrane thickness and lipid orientation can be tuned through the addition of phosphatidylcholine lipids. The hybrid membranes can be fluorescently labelled by incorporating Texas-red DHPE, and their charge modified by incorporating phosphatidylserine and phosphatidylglycerol. By using fluorescein labeled dextran as an example, it is demonstrated that small molecules can be encapsulated into these hybrid liposomes.

\section{Introduction}

Target-oriented drug delivery is one of the biggest challenges in modern drug development. The idea of using carriers to transport and release drug molecules at specific locations in the body is intriguing, and can significantly increase the drug's efficiency and reduce potential side effects. ${ }^{[1-4]}$ Hydrogels and synthetic liposomes are two common attempts to address this problem. ${ }^{[5,6]}$ Despite their success, these non-endogenous drug carriers raise numerous challenges as their efficiency is often limited by the host's immune response $\mathrm{e}^{[6]}$ or requires costly implants. ${ }^{[5]}$

First proposed by Ihler et. al., ${ }^{[7]}$ Red blood cells (RBCs) have been in focus as potential drug carriers. The evident advantage of using RBCs is an extended natural lifespan of these cells within the body, a greater biocompatibility, and a direct access to numerous target sites. ${ }^{[8]}$ With glucocorticoid analogue dexamethasone loaded RBCs, the first RBC-based therapy reached the clinical stage ${ }^{[9]}$ as treatment of Ataxia-telangiectasia, a rare neurodegenerative disease. ${ }^{[10]}$ The two common approaches described in the literature aim to encapsulate drugs and molecules within erythrocyte ghosts or attach reactive agents to RBCs. ${ }^{[11-13]}$ For instance, Thrombomodulin ${ }^{[12]}$ and plasminogen activators ${ }^{[11,13]}$ have been successfully linked to RBCs resulting in an increased circulation time of these molecules. ${ }^{[11-14]}$ Escherichia coli L-asparaginase loaded erythrocytes have been reported to show a ten-fold increase of the pharmacodynamic of this therapeutic enzyme in mice. ${ }^{[15]}$ Although RBCs have numerous advantages over the aforementioned synthetic drug carriers, loaded RBCs or RBC ghosts typically lack specificity with respect to target sites or show a reduced biocompatibility. ${ }^{[16,17]}$ To address these difficulties, recent approaches include hybrid RBC liposomes as drug carriers, ${ }^{[18]}$ or combined membranes from multiple endogenous cells. ${ }^{[19,20]}$

In this paper, we describe the preparation of hybrid erythrocyte liposomes and study the effect of different lipid classes (PC, PS, and PG), as well as varying degrees of saturation and tail length $(14: 0,16: 0-18: 1)$, on the material properties of RBC membranes. First, empty RBC liposomes, often referred to as RBC ghosts, were mixed with synthetic liposomes, sonicated 


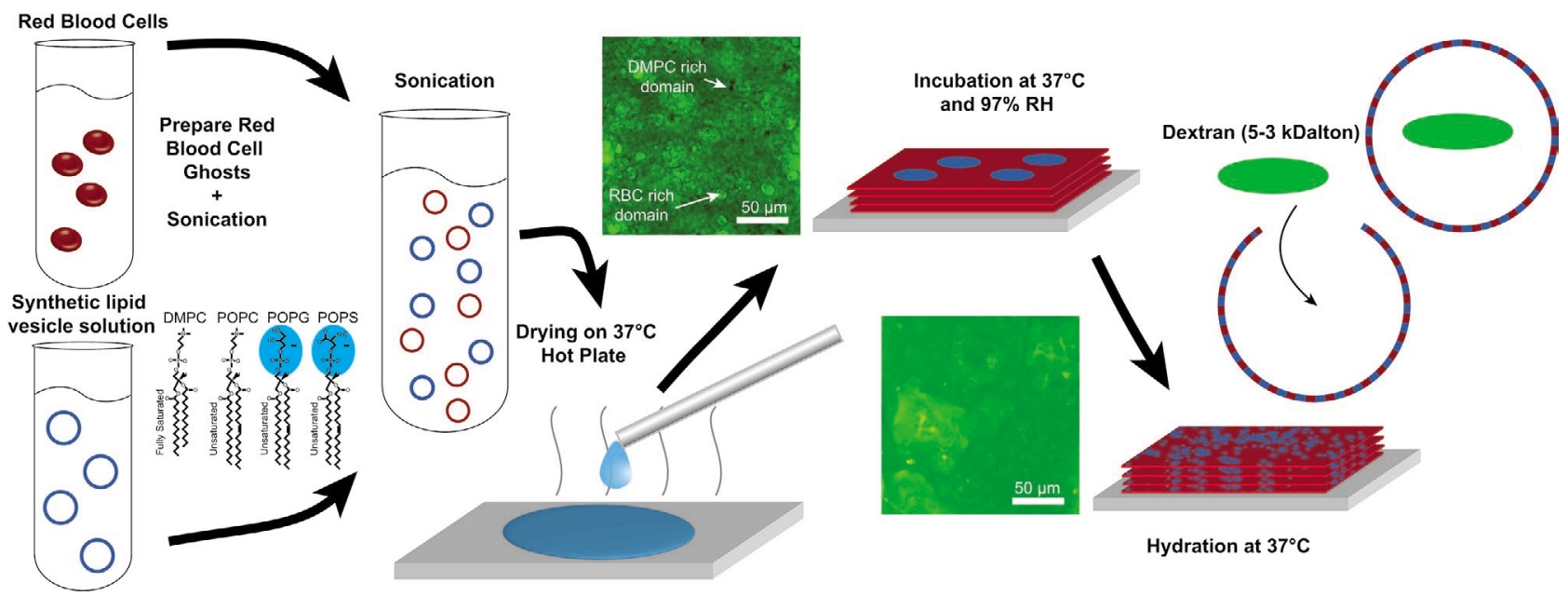

Figure 1. Erythrocyte liposomes (ghosts) are prepared from human RBCs and aqueous stock solutions of synthetic lipid molecules (DMPC, POPC, POPS, and POPG) are prepared. Blood and lipid solutions are sonicated before mixing in ratios of 1:4, 2:3, 1:1 3:2, 4:1, and 9:1. The resulting solution is sonicated 10 times in pulses of $55 \mathrm{~s}$ and applied onto a silicon wafer and allowed to dry and incubate. The images show epi-fluorescent microscopic images of the wafer surface. Green areas correspond to protein-rich, erythrocyte membrane rich areas. Uniform membranes form after incubation. The wafer is then immersed in a solution containing small molecules, which are encapsulated when hybrid erythrocyte liposomes form. In this work, fluorescein labeled dextran was incorporated.

and dried on a solid support. This 2D confinement promotes the fusion of both membrane species during the subsequent incubation. Hybrid liposomes were formed by re-hydrating the dry membranes. Small molecules can be incorporated within these hybrid liposomes during this step. A sketch of the preparation protocol is shown in Figure 1.

Molecular level structural and dynamical information was obtained using X-ray diffraction, epi-fluorescent microscopy, dynamic light scattering (DLS), Zeta potential, UV-vis spectroscopy, and Molecular Dynamics (MD) simulations. Dimyristoylphoshatidylcholine (DMPC), for instance, was found to increase the degree of order while decreasing the membrane thickness. In contrast, palmitoyloleoylglycerophosphocholine (POPC) lowers the overall bilayer thickness and reduces the degree of order. Anionic lipids, such as palmitoyloleoylglycerophosphoserine (POPS) and palmitoyloleoylglycerophosphoglycerol) (POPG), were used to alter the membranes' charge and result in a decreased Zetapotential. On microscopic scales, synthetic lipid molecules fuse homogeneously with erythrocyte membranes when within their solubility limits. However, MD simulations, indicate the presence of dynamic nanometer sized erythrocyte rich and erythrocyte poor domains, mimicking rafts in biological plasma membranes.

\section{Results}

\subsection{Molecular Structure of Hybrid Membranes}

The molecular structure of the hybrid erythrocyte membranes was determined by X-ray diffraction. The measurements were performed on solid supported membranes after incubation but before liposomal fabrication. Scans were done in a humidity and temperature controlled chamber. The setup is depicted in Figure $2 \mathrm{a}$. Figure $2 \mathrm{~b}$ shows $2 \mathrm{D}$ X-ray intensity maps for erythrocyte membranes containing DMPC at ratios of (RBC:DMPC) 1:4, 1:1, and 9:1. Pure RBC membranes are shown as reference.
The observed Bragg peaks are the result of the membrane stacking. The 1:4 sample mainly consists of synthetic DMPC and produces a well pronounced series of peaks. The intensity and number of these peaks decrease as the RBC concentration increases, indicative of a less-well ordered lamellar phase with increased mosaicity. Line-cuts along $q_{\|}=0$ were calculated by integration along the marked rectangle and are shown in Figure 2c. The lamellar spacing, $d_{z}$, was determined from the distance of the reflectivity Bragg peaks using Bragg's law, $d_{z}=2 \pi / q_{z}$.

Electron densities perpendicular to the membranes are presented in Figure 2d, as calculated from a 1D Fourier analysis. The data show an increased electron density around $|z| \approx 20 \AA$, corresponding to the electron-rich head groups of the lipid molecules, and a decreased density in the center of the bilayer $(|z|=0 \AA)$. The membrane thickness was determined by the distance between the two maxima in the electron density profile, and will be referred to as head-to-head distance $d_{H H}$. Figure $2 \mathrm{e}$ shows the result of an angular integration along the dotted line in Figure 2b. A small width in the angular distribution is indicative of well-ordered membranes within the stack. The degree of order is quantified by fitting Herman's orientation function, as detailed in the Experimental Section. A degree of orientation between $\approx 82 \%$ and $\approx 97 \%$ was determined, in good agreement with previous studies on pure red blood cells, ${ }^{[21]}$ and on monoor multicomponent synthetic membranes. ${ }^{[22]}$

Figure 3a compares the reflectivity of different types of synthetic lipids, all at a ratio of 9:1. While the inclusion of DMPC and POPC was found to lead to well organized membranes, the addition of POPS and POPG significantly suppressed higher order peaks, indicative of increased disorder. The corresponding electron densities are shown in Figure S1, Supporting Information, and are in agreement with increasing tail disorder between DMPC-POPC-POPS (no electron density could be determined for the case of POPG because of the absence of higher order Bragg peaks). 


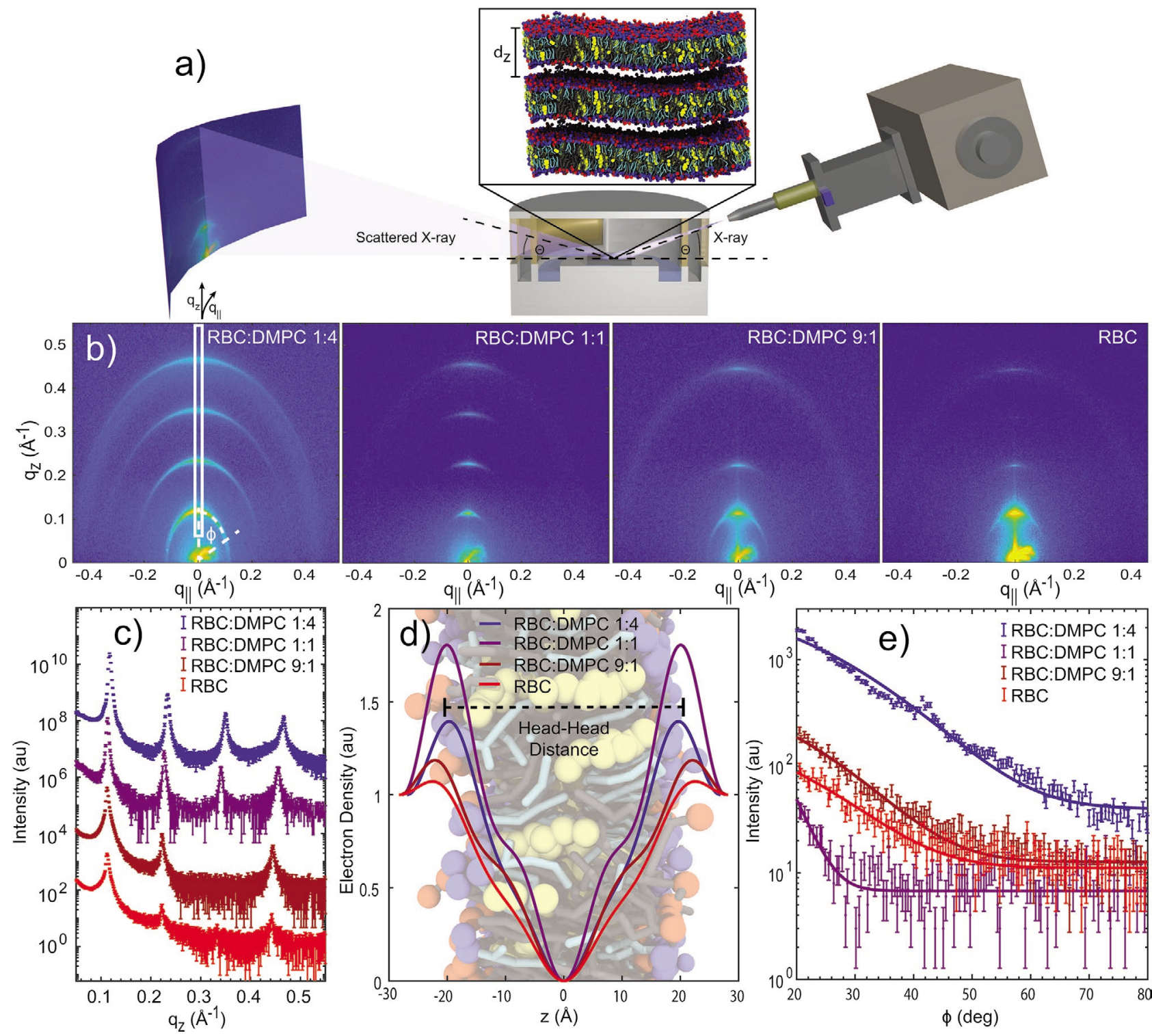

Figure 2. a) Experimental setup. The solid supported membranes are placed in a humidity controlled chamber at $88 \%$ relative humidity. The sample remains horizontal throughout the measurement. $q_{z}$ measures out-of-plane structure while $q_{\|}$measures in-plane structure. b) 2D X-ray intensity maps for three selected RBC:DMPC hybrid samples: 1:4, 1:1, and 9:1. The scattering pattern of pure RBC membranes are included as reference. c) Bright prominent spots are apparent, which appear as series of Bragg-peaks in a line-cut along $q_{z}$ at $q_{\|}=0$ and are result of the lamellar spacing between the stacked membranes. d) Shows the corresponding electron density data determined by a ID Fourier Analysis. The head groups show up as increased electron density around $|z| \approx 20 \AA$. e) The degree of orientation was determined by fitting a Gaussian profile to the scattered intensity along the meridional angle $\phi$ and using Herman's orientation function.

DMPC, POPC, and POPS show a gradual increase in lamellar spacing and head-head distance with an increasing fraction of RBC membranes, as shown in Figures 3b,c. It converges to $\approx 56 \AA$ for the $d_{z}$-spacing and $\approx 44 \AA$ for the head-head distance, in good agreement with previously published measurements on red-blood cell membranes. ${ }^{[21]}$

Two regimes were observed for all lipid species: a linearly increasing regime and a plateau region for higher fractions of $\mathrm{RBC}$ membranes. The latter one indicates minor structural differences as compared to a pure erythrocyte membrane. Consequently, we define the solubility limit as the boundary between both regimes. It was determined for all lipid species by fitting lines to both regimes and determining the $x$-coordinate of the intersect. The fits are indicated in Figure $3 \mathrm{~b}$ by dotted lines using POPC as an example. The solubility limits are plotted in Figure $3 \mathrm{~d}$.

Figure 3e compares the membranes' orientation as function of the synthetic lipid concentration and the lipid species. The highest degree of orientation was observed for an equal ratio between erythrocyte and synthetic lipids, while a high concentration of synthetic lipids causes an overall lower degree of orientation. Values for DMPC are significantly higher as compared 

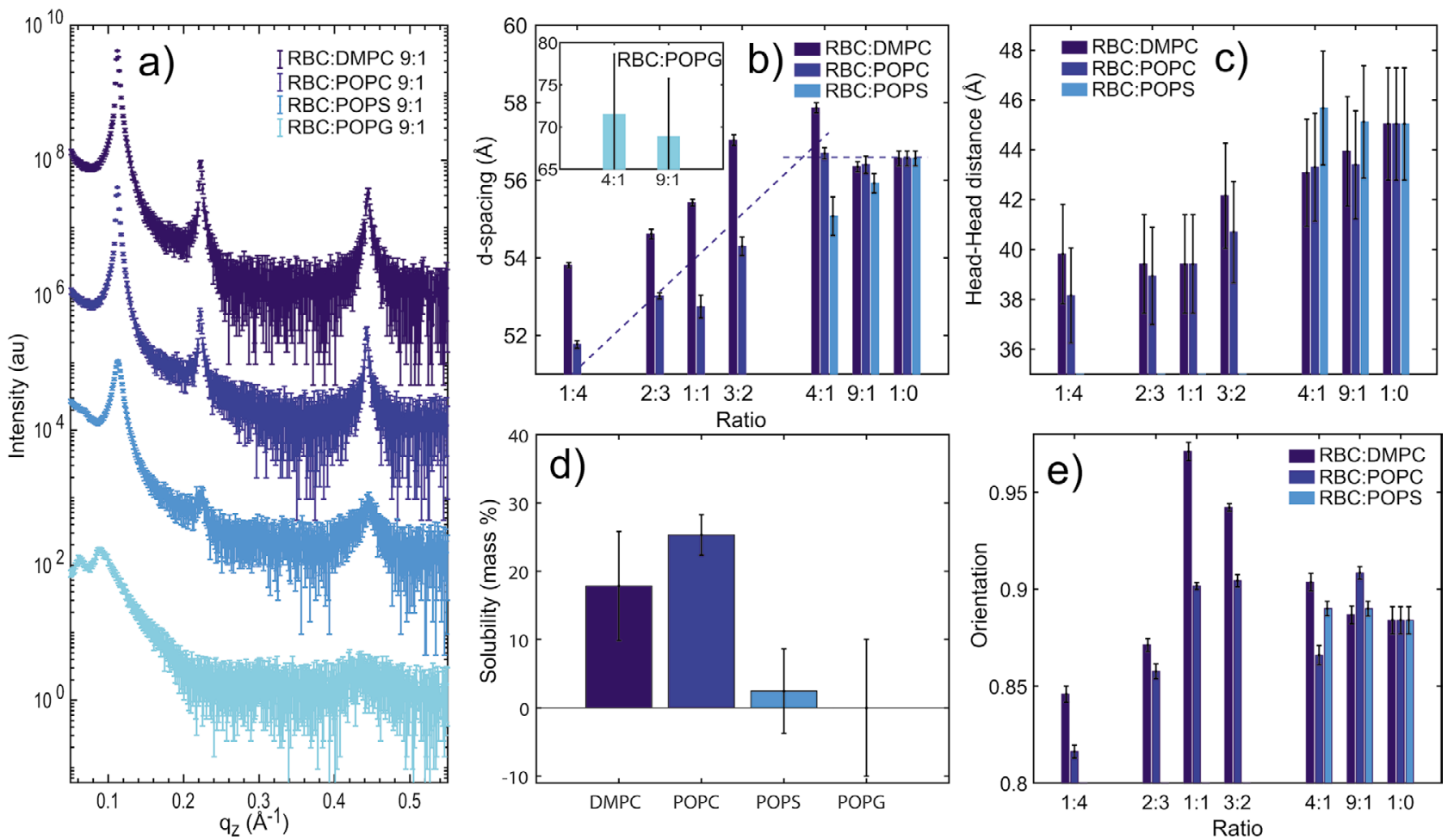

Figure 3. a) Diffraction along $q_{z}$ for hybrid membranes containing 10 mass percent synthetic lipids (DMPC, POPC, POPS, and POPG). b) Lamellar spacing $d_{z}$ as function of synthetic lipid concentration for the different lipid species. c) Corresponding head-head distance as function of the synthetic lipid concentration. d) The solubility limit was determined by the intercept of two linear regimes fitted to part b). The fits for POPC are shown as dotted lines. e) shows the degree of order as a function of the synthetic lipid concentration and species, as determined by Herman's orientation function.

to POPC and POPS, as fully saturated lipid molecules seem to induce additional order in the erythrocyte membrane.

\subsection{Molecular Dynamics Simulations}

Experiments point to a homogeneous mixing of erythrocyte and synthetic membranes. MD simulations give information about the underlying dynamical processes. Coarse grained MD simulations were performed using erythrocyte membranes containing $10 \mathrm{~mol} \%$ ( $11.5 \mathrm{mass} \%), 50 \mathrm{~mol} \%$ ( $\approx 54 \mathrm{mass} \%)$ and $80 \mathrm{~mol} \%$ ( $\approx 82.4$ mass\%) DMPC to study the dynamics and in-plane organization of the hybrid membranes. Snapshots after $5 \mu \mathrm{s}$ of simulation time are depicted in Figure 4a. Red and blue spheres represent the phosphate groups of lipid molecules assigned to the red blood cell fraction and synthetic DMPC lipids, respectively. Cholesterol is depicted by yellow spheres. The snapshots indicate a homogeneous mixture of both membrane species. This changes when comparing the time averaged density of the lipid molecules, as shown in Figure 4b, where dynamic small, nanometer-sized patches become visible.

The size of the erythrocyte and DMPC patches can be determined by counting the number of pixels above the median density multiplied with the resolution. Patch sizes ranging from 35.3 to $147.0 \mathrm{~nm}^{2}$ were determined, as listed in Table 1 . These patches are dynamic entities and undergo molecular fluctua- tions, which mimic the natural fluctuations observed in biological plasma membranes. Simulation videos are provided in videos S1-S3, Supporting Information, over the course of $2 \mu \mathrm{s}$.

\subsection{Liposome Characterization and Encapsulation of Molecules}

Fluorescently labeled hybrid liposomes (Figure 5a) were visualized using an epifluorescent microscope (Figure 5b), as detailed in the Experimental Section. Liposomes had a typical size of $10-15 \mu \mathrm{m}$ and varied in shape, as shown in Figure 5c. The membrane appears as a bright red edge with a width of $\approx 550 \mathrm{~nm}$, within the resolution limit of the microscopic setup used. The liposomes in Figure 5c appear to be homogeneously labeled and show no indication of phase separation or domain formation within the resolution limit of the microscope.

Size and size distribution of all hybrid liposomes were determined using DLS and are listed in Table 2. Liposomes were sonicated before the measurements for better comparison. The average diameter ranges from $\approx 120$ to $\approx 208 \mathrm{~nm}$. While pure RBC liposomes (ghosts) showed an average size of $\approx 200 \mathrm{~nm}$, inclusion of saturated and charged lipids resulted in smaller sized liposomes. The inclusion of dextran led to a small increase of the liposome size. While the size distribution of pure RBC liposomes was found to be reasonably well defined, as indicated by the polydispersity index, the inclusion of synthetic lipids increased the size distribution in all cases. 


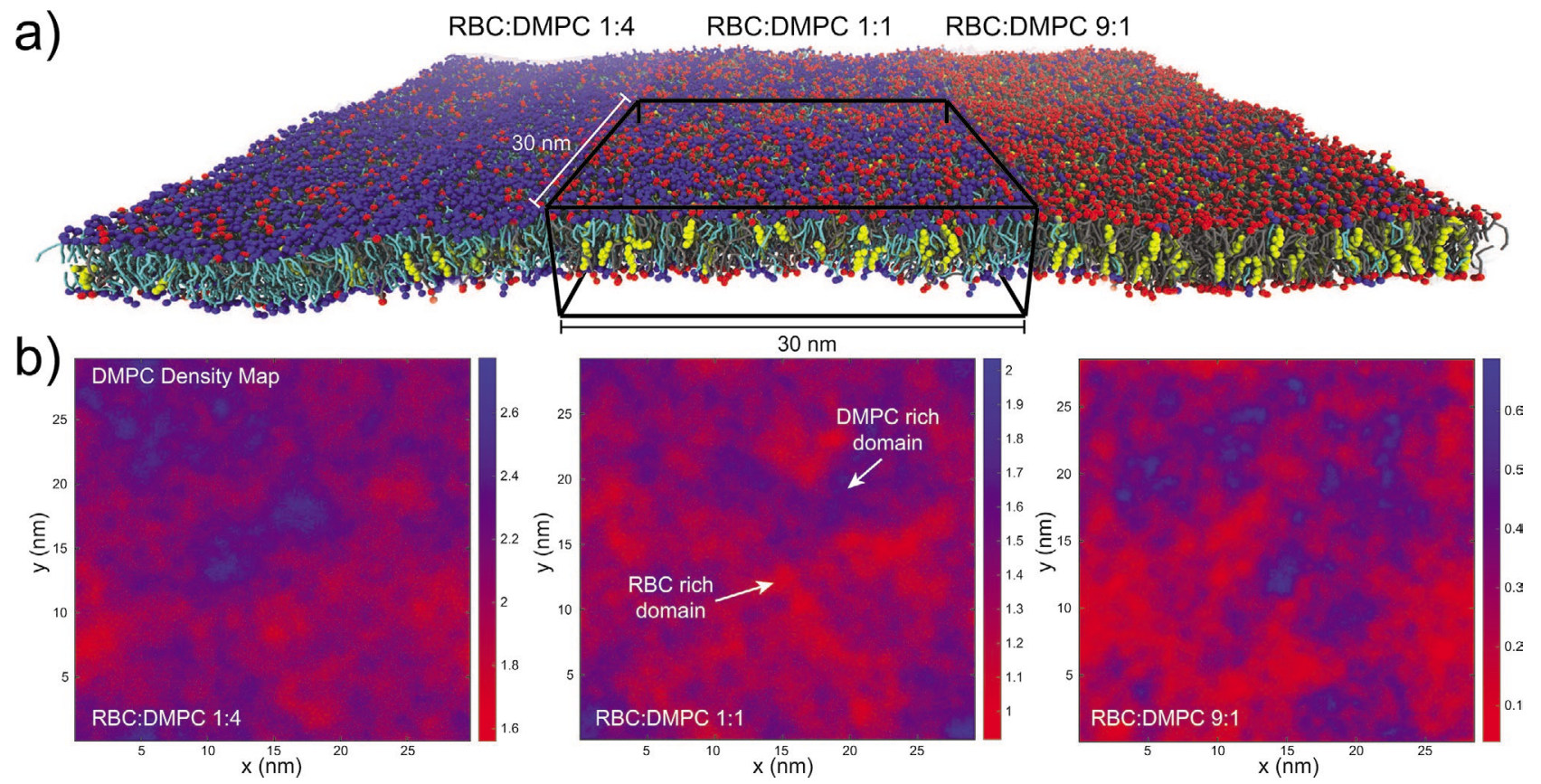

Figure 4. a) Snapshot of MD simulation at $t=5 \mu \mathrm{s}$. The phosphate group is indicated by red and blue spheres, corresponding to RBC and DMPC lipids respectively. Cholesterol is represented by yellow spheres. Gray and light blue lines represent the lipid tails of RBC and DMPC lipids respectively. The simulation box is shown as a black box and measures $30 \times 30 \mathrm{~nm}$. Water molecules are omitted for clarity. b) DMPC density maps for hybrid membranes containing 80,50, and 10 mass\% DMPC, respectively. Blue color indicates a high concentration of DMPC while the red regions correspond to a DMPC depletion, that is, a high concentration of RBC lipid species.

DMPC and POPC resulted in a significant broadening of the distribution while adding charged lipids (POPS and POPG) increased the distribution moderately, only. The Zeta-potential was determined for pure RBC ghosts as well as for hybrid membranes containing 10\% and 20\% POPS and POPG. By applying an alternating current, the Zeta-potential is a measurement of potential charge-dependent dynamics of the liposomes, as depicted in Figure 6a. Both anionic lipids decrease the Zetapotential, as shown in Figure 6b. While erythrocyte membranes show a Zeta-potential of $-25.7 \pm 5 \mathrm{mV}$, POPS and POPG were found to lower the potential to $-35.5 \pm 5 \mathrm{mV}$ and $-47.5 \pm 5 \mathrm{mV}$, respectively. Previous studies ${ }^{[23]}$ have reported a linear relationship between the concentration of charged lipid molecules and the Zeta-potential. However, our data show no concentration dependence, within statistical errors.

Molecules were encapsulated in the hybrid liposomes by hydrating the solid supported membranes in an aqueous solution, as pictured in Figure 7a. The experimental setup is shown in Figure $7 b$. Figure $7 c$ shows liposomes that were prepared

Table 1. DMPC domain sizes were determined from 2-dimensional density maps by counting the number of pixels above a threshold and multiplying the results by the resolution.

\begin{tabular}{lcc}
\hline Membrane System & \multicolumn{2}{c}{ DMPC domain size $\left[\mathrm{nm}^{2}\right]$} \\
\cline { 2 - 3 } & upper leaflet & lower leaflet \\
\hline RBC:DMPC 1:4 & 75.4 & 95.2 \\
RBC:DMPC 1:1 & 46.1 & 147.0 \\
RBC:DMPC 9:1 & 79.0 & 35.3 \\
\hline
\end{tabular}

in a $1 \mathrm{mg} \mathrm{mL} \mathrm{m}^{-1}$ solution of $3-5 \mathrm{kDa}$ fluorescein labeled dextran. The interior of the liposomes lights up in green under the microscope indicating that the dextran is located within the hybrid liposomes. Dextran has been previously reported to interact with the $\mathrm{RBC}$ membrane at larger concentration of dextran $\left(>10 \mathrm{mg} \mathrm{mL}^{-1}\right) \cdot{ }^{[24-26]}$ The homogeneous color and intensity of the liposomes, however, indicate that the molecules are homogenously distributed within the liposomes (within the resolution limit of the microscope).

\section{Discussion}

Endogenous substances can transport drugs hidden from the immune system and allow the design of far more complex liposomes. However, controlling membrane morphology and structure is essential in generating applicable carrier systems. Recent papers ${ }^{[18-20]}$ used a combination of biological and synthetic membranes. Human erythrocytes are well suited as a base for such hybrid liposomes as their membrane can be easily isolated from other cellular components. At the same time they have the potential of minimizing immune reactions and circulating in the blood stream for extended periods of time.

The protocol presented in this work allows the efficient preparation of hybrid erythrocyte membranes. Our data indicate that synthetic lipid molecules can be incorporated into $\mathrm{RBC}$ membranes and show no indication of phase separation, in contrast to previously published protocols. ${ }^{[18]}$ The crucial intermediate step in the protocol is the preparation of solid supported hybrid membranes. The $2 \mathrm{D}$ confinement together with 


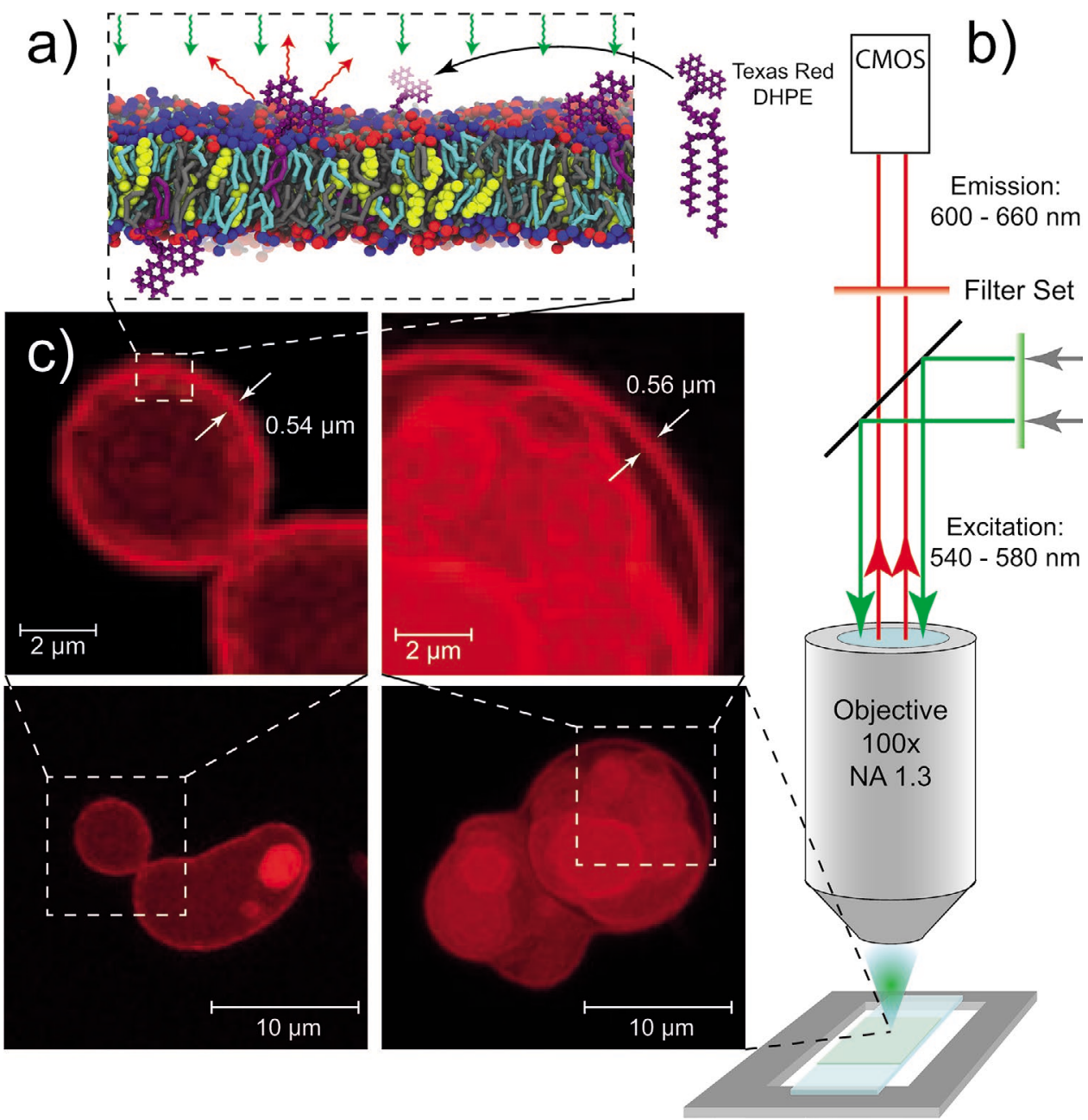

Figure 5. a) Liposomes containing 0.5 mass\% head-group labeled Texas red DHPE were prepared. b) The Epi-fluorescent microscope uses an excitation filter of 540-580 nm and an emission filter of 600-660 nm. c) The membrane of the liposomes shows up as a bright, red barrier under the microscope. Complex liposome structures were observed consisting of multiple fused spherical objects. The membrane thickness was found to be $560 \mathrm{~nm}$, the resolution limit of the setup.

Table 2. The diameter of the hybrid liposomes after sonication determined by dynamic light scattering (DLS). The average diameter ranges from $\approx 120 \mathrm{~nm}$ to $\approx 208 \mathrm{~nm}$. Inclusion of saturated and charged lipids resulted in smaller sized liposomes. The inclusion of dextran led to a small increase of the liposome size. While the size distribution of pure RBC liposomes is reasonably well defined, as indicated by the polydispersity index, the inclusion of synthetic lipids increased the size distribution in all cases. DMPC and POPC resulted in a significant broadening of the distribution while adding charged lipids (POPS and POPG) increased the distribution moderately, only.

\begin{tabular}{lcc}
\hline Liposomes & Diameter [nm] & Polydispersity index \\
\hline pure ghosts & $199.05 \pm 3.34$ & $0.14 \pm 0.013$ \\
RBC:DMPC 4:1 & $147.8 \pm 2.3$ & $0.32 \pm 0.04$ \\
RBC:DMPC 9:1 & $198.9 \pm 12.1$ & $0.42 \pm 0.06$ \\
RBC:POPC 4:1 & $208.0 \pm 8.8$ & $0.48 \pm 0.079$ \\
RBC:POPC 9:1 & $174.5 \pm 6.7$ & $0.46 \pm 0.01$ \\
RBC:POPS 4:1 & $164.3 \pm 1.0$ & $0.174 \pm 0.02$ \\
RBC:POPS 9:1 & $147.9 \pm 1.35$ & $0.218 \pm 0.004$ \\
RBC:POPG 4:1 & $138.3 \pm 1.6$ & $0.241 \pm 0.003$ \\
RBC:POPG 9:1 & $147.2 \pm 1.1$ & $0.248 \pm 0.008$ \\
RBC:DMPC 4:1 with Dextran & $160.0 \pm 60$ & $0.3 \pm 0.08$ \\
RBC:POPC 4:1 with Dextran & $119.3 \pm 1.46$ & $0.245 \pm 0.01$
\end{tabular}




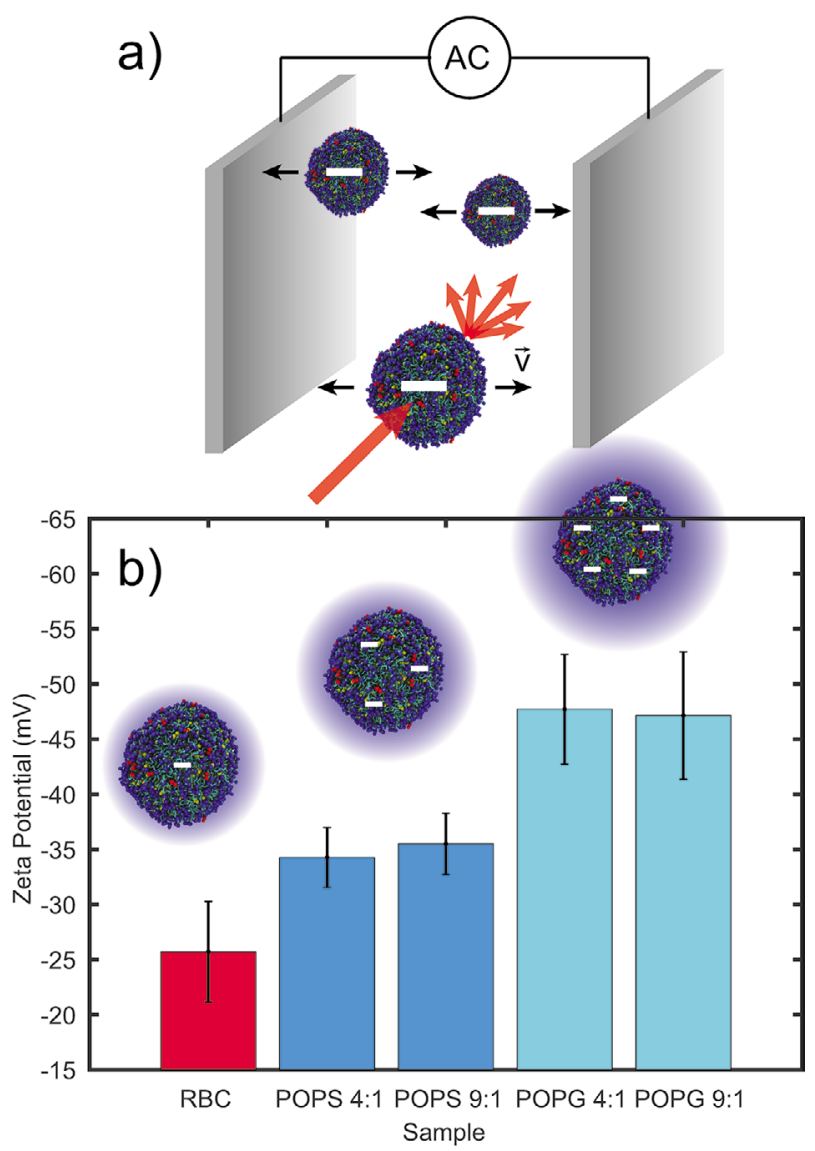

Figure 6. a) The Zeta-potential of the hybrid liposomes was determined using a Zetasizer Nano ZS from Malvern Panalytical. Alternating current is applied via two electrodes allowing the measurement of chargedependent dynamics of the liposomes. b) Zeta-potential for RBC ghosts and hybrid liposomes containing 10 and 20 mass\% POPS and POPG, respectively. Both synthetic lipid species add additional charge to the membrane.

drying and incubation promotes the fusion of both membrane species. While microscopy is a very efficient tool to determine the topology of hybrid membranes and liposomes, X-ray diffraction gives access to nanoscale bulk information. Experiments were complemented by computer simulations, which can now model plasma membranes realistically, ${ }^{[27]}$ and provide important information on nanoscopic dynamics and mixing.

The lack of split peaks in the X-ray diffraction measurements, together with the homogeneous red color of the fluorescently labeled hybrid liposomes indicate a homogenous fusion of both membrane species. A deeper insight into the mixing is provided by MD simulations. While snapshots of the simulation indicate a uniform mixture of erythrocyte and synthetic membranes, the time averaged density maps show evidence for dynamic nanometer sized patches of erythrocyte rich and poor regions.

Previous MD simulations on large-scale models show a similar de-mixing of lipid species in biological cell membranes. Ingolfsson and co-authors also demonstrated that these patches form and disappear on nano to microsecond time scales. ${ }^{[27]}$ It is now widely accepted that local fluctuations

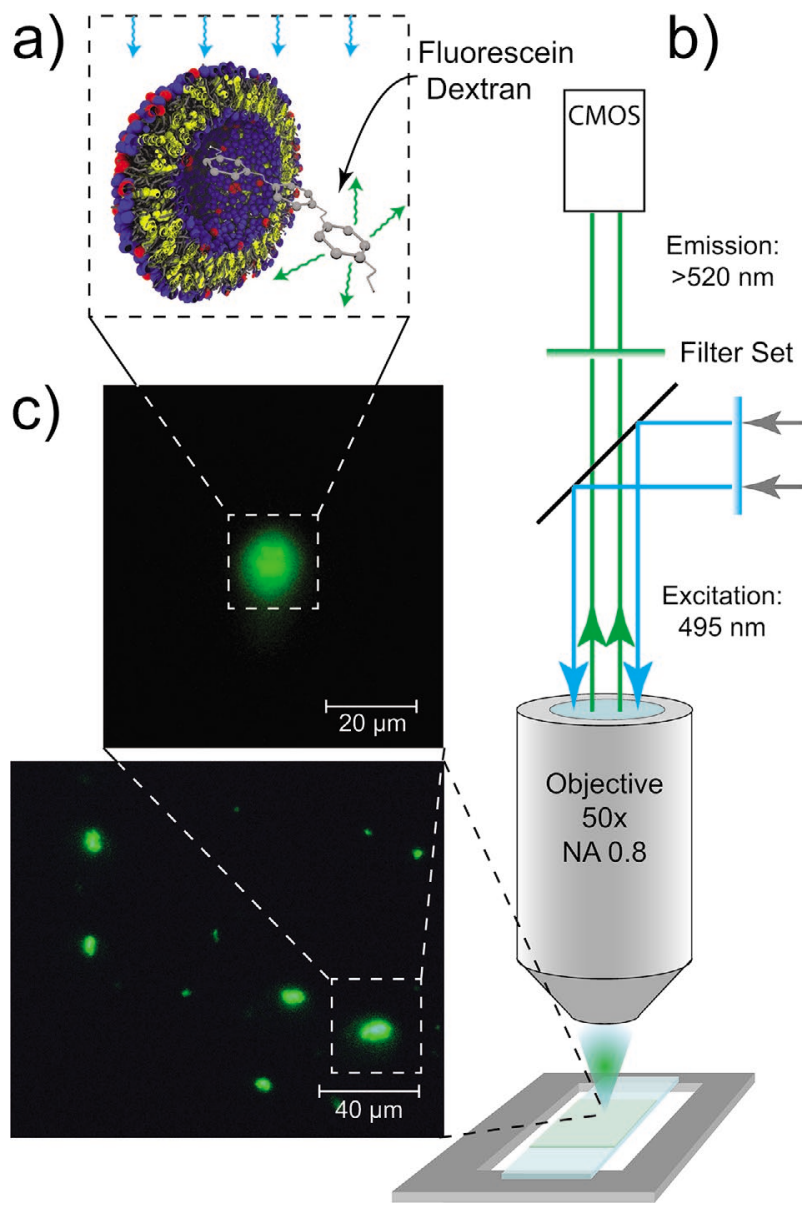

Figure 7. a) The hybrid liposomes were filled with fluorescein labeled dextran. b) The experimental setup consisted of an epi-fluorescent microscope with an excitation wavelength of $495 \mathrm{~nm}$ and a long-pass analysis filter with a barrier wavelength of $520 \mathrm{~nm} . \approx 20 \mu \mathrm{L}$ of the liposome solution was applied to a microscope slide and sealed by a cover slip. c) Epifluorescent microscopic image of the prepared liposomes, which light up as bright green circles on the image.

are an intrinsic property of membranes. ${ }^{[27-31]}$ These fluctuations typically average out on longer length and time scales leading to a uniform membrane structure. The observed small dynamic domains are, therefore, not the result of a static phase separation between both membrane species but the result of nanoscopic molecular fluctuations, typically observed in biological membranes.

We note that erythrocyte membranes are in general asymmetric. ${ }^{[32]}$ POPS, for instance, is exclusively found in the inner leaflet of mammalian cell membranes. PE lipids are located on both leaflets with a preferred position on the inner leaflet. In experiments, RBC ghosts in general co-exist in inside-out and right-side out configurations, as discussed, for example, in ref. [33]. When the ghosts are dried out on the solid support there is a random stacking of the different membrane orientations and the resulting membrane layer and liposomes are on average symmetric with respect to the composition of the inner and outer leaflets. We can, therefore, not conclude on effects due to potential asymmetry of the hybrid membranes and liposomes. MD models were prepared by first modeling an 
asymmetric RBC membrane. Synthetic lipids were then equally added to to both leaflets to mimic the experimental conditions. However, there was no evidence for an asymmetric distribution of the synthetic lipids between the two leaflets within the $5 \mu \mathrm{s}$ of simulation time, which could for instance be caused by lipid flip-flop. We therefore assume a stable symmetric distribution of synthetic lipids across the leaflets.

While up to 30 mass\% DMPC and POPC can be mixed with erythrocyte membranes, small amounts of charged lipids disturb the membrane assembly resulting in decreased (POPS) and increased (POPG) lamellar spacings, and the formation of smaller liposomes. In particular POPG inhibits the assembly of stacked membranes. These findings are supported by the DLS and Zeta-potential measurements. Inclusion of synthetic lipids (except for RBC:POPC 4:1) resulted in smaller sized liposomes, indicating a reduced stability. Inclusion of dextran led to a small increase of the liposome size.

While the Zeta-potential is not a direct measure of the charge density, as detailed in the paper by Bhattacharjee, ${ }^{[34]}$ it determines the interfacing potential of the so-called slipping plane around the liposome. The Zeta-potential of the erythrocyte membrane was measured to be $-25.7 \pm 4.6 \mathrm{mV}$, in good agreement with previous studies. ${ }^{[35]}$ POPS and POPG further decrease the Zeta-potential to $-34.3 \pm 2.7$ and $-35.5 \pm 2.8 \mathrm{mV}$, and $-47.7 \pm 5$ and $-47.1 \pm 5.8 \mathrm{mV}$, respectively, indicating an increased negative membrane charge. Previous studies reported a concentration-dependent decrease of the Zeta-potential in the presence of $\mathrm{PS}^{[23]}$ and $\mathrm{PG}^{[35]}$ lipids in synthetic membranes. The concentration dependent differences in our measurements, however, are within the statistical errors.

Liposomes can be loaded with molecules during the rehydration phase, when the molecule containing solution is applied onto the dried out supported membranes. The molecules are then encapsulated when the membranes re-hydrate and liposomes form. The loading efficiency is defined as the amount of encapsulated molecules relative to the initial concentration of the molecule, and was determined using UV-vis spectroscopy. We determine an encapsulation efficiency of $2.1 \pm 0.7 \%$ for dextran in hybrid liposomes containing 20\% DMPC and $3.5 \pm 0.5 \%$ for hybrid liposomes containing 20\% POPG. Both efficiencies are comparable, in the order of a few percent. While these efficiencies are only slightly smaller than typical loading efficiencies reported for synthetic liposomes of $\leq 10 \%,{ }^{[36]}$ they can likely be increased with an optimized protocol in the future.

The biocompatibility of RBC based drug delivery systems is a long standing concern. While the hybrid membranes are entirely composed of biocompatible materials, ${ }^{[18]}$ previous studies have shown that loading of RBCs can have a significant impact on their biocompatibiliy. ${ }^{[16,17]}$ This is, however, often a result of heavy modifications to the RBC surface. The longevity of modified RBC depends on numerous mechanisms and has been studied in detail. ${ }^{[37-40]}$ However, a key factor for the biocompatibility is the resilience of the hybrid liposomes against mechanical stress. Figure S5, Supporting Information, shows the result of a lysis assay where hybrid liposomes containing 20\% DMPC were exposed to increasing osmotic stress by altering the molar concentration of a phosphate buffer saline. This method has previously been described as in vitro test for biocompatibiliy of RBC membranes. ${ }^{[41]}$ The data show an increase of lysed hybrid liposomes below $5.6 \mathrm{mM}$ phosphate buffer saline, corresponding to $\mathrm{a} \approx 150 \mathrm{mOsm}$ solution, in good agreement with results for pure $\mathrm{RBC}^{\left[{ }^{[41]}\right.}$ It can, therefore, be expected that the hybrid liposomes will behave similar to RBCs in vivo. Although these results prove the biocompatible nature of these hybrid liposomes, the compatibility and longevity of the drug delivery system will be confirmed in animal studies in the future.

\section{Conclusion}

In summary, we prepared hybrid erythrocyte membranes by purifying and doping endogenous RBC bilayers with synthetic lipid molecules. We studied the impact of different lipid classes (PC, PS, and PG), as well as different tail saturation (14:0, 16:0 18:1), on membrane morphology and structure using X-ray diffraction, MD simulations, dynamic light scattering, Zeta potential, UV-vis spectroscopy, and epi-fluorescent microscopy. Fluorescently labeled hybrid liposomes were prepared using Texas-red DHPE (TR-DHPE) and fluorescein labeled dextran.

Different synthetic lipid species functionalize the RBC membranes by altering their thickness, order and the surface charge. As a fully saturated lipid, DMPC was found to induce additional order, while POPC led to a more disordered bilayer with increased mosaicity. Both lipids result in a significantly reduced membrane thickness. The addition of POPS and POPG led to the formation of charged liposomes, as proven by a decreased Zeta-potential.

Experimental and computational findings indicate a homogenous mixing of erythrocyte and synthetic membranes down to the nanoscale. Formation of dynamic nanometer sized patches of constantly mixing and de-mixing erythrocyte rich and poor domains was, however, observed as a result of molecular fluctuations. By using dextran as an example, we show that small molecules can be encapsulated into the hybrid liposomes.

\section{Experimental Section}

This research was approved by the Hamilton Integrated Research Ethics Board (HIREB) under approval number 1354-T. Informed consent was obtained from all blood donors. The authors confirm that all methods were performed in accordance with the relevant guidelines and regulations.

Preparation of Hybrid Membrane Mixtures: The preparation is based on a protocol first published by Himbert et al.[21] All blood samples were collected using sodium heparin coated venous blood collection tubes from BD (Product Number: BD 367874). The blood was washed twice and the RBC were isolated as described in ref. [21]. Hemolysis was induced by adding $50 \mu \mathrm{L}$ of the hematocrit to $1 \mathrm{~mL}$ of a diluted buffer solution in a $1.5 \mathrm{~mL}$ reaction tube. This buffer is prepared by mixing $16 \mathrm{~mL}$ of Phosphate Buffered Saline (PBS) with $484 \mathrm{~mL}$ of $18 \mathrm{M} \Omega \mathrm{cm}$ ultra pure water and adjusting the $\mathrm{pH}$ to a value of 8 by slowly adding potassium hydroxide. The reaction tubes were immediately stored on ice to prevent a fast re-closing of the ruptured cells. This enables the removal of hemoglobin and other cellular compartments using multiple washing steps as demonstrated in ref. [21]. The protocol results in a white pellet containing empty RBC liposomes. The pellets from 24 reaction tubes were combined and the volume was adjusted to $0.5 \mathrm{~mL}$ resulting in a ghosts concentration of $\approx 14 \mathrm{mg} \mathrm{mL}^{-1}$. $^{[2]]}$ The resulting solution was tip sonicated 20 times for $5 \mathrm{~s}$ each at a power of $100 \mathrm{~W}$. Note, that the reaction tube was placed on ice during sonication to prevent the sample 
from overheating. Afterwards, the tube was centrifuged for $15 \mathrm{~min}$ at $\approx 20000 \mathrm{~g}$. The supernatant consists of a solution of small nanometersized liposomes, ${ }^{[2]]}$ referred to as Blood Solution.

Aqueous solutions of dispersed 1,2-dimyristoyl-sn-glycero-3phoshatidylcholine (DMPC), 1-palmitoyl-2-oleoyl-glycero-3-phosphocholine (POPC), 1-palmitoyl-2-oleoyl-sn-glycero-3-phospho-L-serine (POPS) and 1-palmitoyl-2-oleoyl-sn-glycero-3-phospho-(1'-rac-glycerol) (POPG) were prepared by dissolving $14 \mathrm{mg}$ of each lipid in $1 \mathrm{~mL}$ of $18.2 \mathrm{M} \Omega \mathrm{cm}$ ultra pure water. The resulting solution was tip sonicated 20 times for $10 \mathrm{~s}$ each at a power of $100 \mathrm{~W}$ until the solutions were clear. This sonicated solution will be referred to as Lipid Solution.

For DMPC and POPC, assays at ratios of (Blood Solution:Lipid Solution) 1:4, 2:3, 1:1, 3:2, 4:1, and 9:1 were prepared. For POPS and POPG, both solutions were mixed in ratios of 4:1 and 9:1. The reaction tube with the final solution was placed on ice and tip sonicated 20 times for $5 \mathrm{~s}$ each.

Fluorescently labeled membranes were prepared by doping the bilayers with Texas Red 1,2-Dihexadecanoyl-sn-Glycero-3Phosphoethanolamine (TR-DHPE) (Thermo Fisher, Catalog number: T1395MP) which was used previously as an indicator for liquid disordered $I_{d}$ domains. ${ }^{[42-45]}$ TR-DHPE was reported for its interaction with lipid molecules, such as DPPC, ${ }^{[46]}$ resulting in a reduced diffusion coefficient, ${ }^{[46]}$ and induced domain formation ${ }^{[47]}$ at higher concentrations (>0.2 mol\%). $1 \mathrm{mg}$ of TR-DHPE was dissolved in $1 \mathrm{ml}$ chloroform. $5.6 \mathrm{mg}$ of DMPC (this corresponds to hybrid membranes containing 40 mass\% DMPC) was dissolved in $1 \mathrm{~mL}$ chloroform. $52 \mu \mathrm{L}$ of the TR-DHPE solution was mixed with the DMPC solution in a glass vial. Chloroform was removed by blowing dry $\mathrm{N}_{2}$ gas in the glass vial for $\approx 20$ min before mixing the sample with ultra pure water and tip sonicating 20 times for $10 \mathrm{~s}$ each at a power of $100 \mathrm{~W}$. The concentration of TR-DHPE corresponds to $0.5 \mathrm{~mol} \%$ in the Lipid Solution and 0.003 mass\% when mixed with erythrocyte membranes. This solution will be referred to as Fluorescent Solution.

For the stained membrane assays, the Fluorescent Solution was mixed with $80 \mu \mathrm{L}$ of the Blood Solution creating a 3:2 sample.

Preparation of Liposomes: The preparation of liposomes is a two-step process. First, erythrocyte ghosts and synthetic liposomes were mixed and sonicated. The solution was then applied to a surface, slowly dried, and incubated. The resulting solid supported membranes initially show large, micrometer-sized erythrocyte, and synthetic domains as depicted in Figure 1. During incubation, the large domains merge to form homogenous hybrid membranes.

Membranes were applied onto single-side polished silicon wafers. $100 \mathrm{~mm}$ diameter, $300 \mu \mathrm{m}$ thick silicon wafers were pre-cut into $10 \times 10 \mathrm{~mm}^{2}$ chips. The wafers were functionalized with a solution of $15 \mathrm{~mL}$ sulfuric acid and $5 \mathrm{~mL}$ hydrogen peroxide (Piranha solution) resulting in a hydrophilic surface. This strong oxidizing agent removes all organic contaminants on the surface, but do not disturb the native silicon oxide layer. Each wafer was then thoroughly rinsed with $\approx 50 \mathrm{~mL}$ of ultra pure water with a resistance of $18.2 \mathrm{M} \Omega \mathrm{cm}$ and placed on a hot plate $\left(37^{\circ} \mathrm{C}\right)$ in a $3 \mathrm{D}$ orbital shaker. $100 \mu \mathrm{L}$ of the hybrid membrane solution was pipetted slowly onto the wafer. The sample was covered with a tilted lid of a petri dish and allowed the membrane solution to slowly dry within $\approx 12 \mathrm{~h}$. The dried wafers were then incubated at $97 \%$ relative humidity and $37{ }^{\circ} \mathrm{C}$ for 3 days by placing the samples in a sealed container with a saturated $\mathrm{K}_{2} \mathrm{SO}_{4}$ solution. This allows the erythrocyte and synthetic membrane domains to fuse into a homogenous membrane phase, as shown in Figure 1. Sample pictures of different RBC-DMPC ratios are shown in Figure S2, Supporting Information.

Liposomes were then synthesized by placing the silicon wafers in a reaction tube filled with $2 \mathrm{~mL}$ of ultra pure water. The tubes were bath sonicated for $1 \mathrm{~h}$ at $37^{\circ} \mathrm{C}$. This re-hydrates the membrane stack and lets the membranes bleb, leaving a blank silicon wafer. The resulting solution had a concentration of $\approx 7 \mathrm{mg} \mathrm{mL}^{-1}$ of membrane material. Liposomes were characterized by dynamic light scattering (DLS) and the determination of the Zeta-potential, as detailed below.

To encapsulate molecules within the liposomes, the solid supported membranes were placed in $2 \mathrm{~mL}$ of an aqueous solution of $1 \mathrm{mg} \mathrm{mL}^{-1}$ fluorescein dextran and bath sonicated at $37^{\circ} \mathrm{C}$ for $\approx 1 \mathrm{~h}$. The sample was then centrifuged for $20 \mathrm{~min}$ at $20000 \mathrm{~g}$. The supernatant was removed and replaced with ultra pure water. This washing step was repeated twice to isolate the stained liposomes. The resulting solution was applied onto a microscope slide and covered with a coverslip prior to imaging.

Liposomes prepared by this protocol showed a large polydispersity index (PDI). Measurements performed on pure RBC liposomes determined a average size of $481.0 \pm 11.41 \mathrm{~nm}$ and a PDI of $0.53 \pm 0.027$. This can be optimized by an additional tip sonication (20 times for $5 \mathrm{~s}$ each at a power of $100 \mathrm{~W}$ ) of the liposomes resulting in a average size of $199.05 \pm 3.34$ with a PDI of $0.14 \pm 0.013$.

$X$-ray Diffraction Experiment: X-ray scattering data was obtained using the Biological Large Angle Diffraction Experiment (BLADE) in the Laboratory for Membrane and Protein Dynamics at McMaster University. BLADE uses a $9 \mathrm{~kW}(45 \mathrm{kV}, 200 \mathrm{~mA}) \mathrm{CuK} \alpha$ rotating anode at a wavelength of $1.5418 \AA$ using a Rigaku HyPix-3000 2D semiconductor detector with an area of $3000 \mathrm{~mm}^{2}$ and $100 \mu \mathrm{m}$ pixel size. ${ }^{[48]}$ All samples were prepared and measured in replicates to check for consistency. Both source and detector are mounted on movable arms such that the membranes stay horizontal during the measurements. Focusing multilayer optics provides a high intensity parallel beam of $\approx 200 \mu \mathrm{m}$ with monochromatic X-ray intensities of up to $10^{8}$ counts. Note that there is no risk of sample damage using this in-house technique because of the relatively low intensity of the X-ray beam as compared to synchrotron sources. The samples were mounted in a custom-built humidity chamber during the experiments to control the humidity of the membranes. The result of an X-ray experiment is a $2 \mathrm{D}$ intensity map of a large area of the reciprocal space, as sketched in Figure 2a,b, covering length scales from about 2.5 to $100 \AA$. All scans were measured at $28{ }^{\circ} \mathrm{C}$ and $88 \%$ relative humidity $(\mathrm{RH})$ hydration. As depicted in Figure $2 \mathrm{a}$, the wafers were oriented in the X-ray diffractometer, such that the $q_{\|}$-axis probed lateral structure, parallel to the wafer surface, and the perpendicular axis, $q_{z}$, probed out-of-plane structure, perpendicular to the substrate.

The out-of-plane structure of the membrane was determined using specular reflectivity. The relative electron density, $\rho(z)$, is approximated by a 1D Fourier analysis. ${ }^{[49]}$

$\rho(z)=\frac{2}{d_{z}} \sum_{n=1}^{N} \sqrt{l_{n} q_{n}} v_{n} \cos \left(\frac{2 \pi n z}{d_{z}}\right)$

where $N$ is the highest order of the Bragg peaks observed. $F\left(q_{n}\right)$ is known as the form factor and is determined by multiplying the integrated peak intensity $I_{n}$ with $q_{n}{ }^{[49]}$ and is in general a complex quantity. In case of centro-symetrie, the form factor becomes real and the phase problem of crystallography, therefore, simplifies to the sign problem $F\left(q_{z}\right)= \pm\left|F\left(q_{z}\right)\right|$. A X-ray diffraction experiment probes the form factor at discrete values of $q_{z}$, and continuous function, $T\left(q_{z}\right)$, can be fitted to the data. ${ }^{[49]}$

$T\left(q_{z}\right)=\sum_{n} \sqrt{I_{n} q_{n}} \operatorname{sinc}\left(\frac{1}{2} d_{z} q_{z}-\pi n\right)$.

Once an analytical expression for $T\left(q_{z}\right)$ has been determined from fitting the experimental peak intensities, the phases $v_{n}$ can be assessed from $T\left(q_{z}\right)$. The phase array $v_{n}=\left[\begin{array}{llll}-1-1 & 1-1 & 1\end{array}\right]$ was used for all samples.

The electron densities determined by Equation (1) are on a relative scale. In order to compare the electron densities in Figure $2 d$ and Figure $\mathrm{S1}$, Supporting Information, $\rho$ in the membrane center at $z=0$ was set to 0 and the electron density at the boundaries, which probe the water layer between the stacked membranes, were scaled to 1 .

To determine the degree of orientation of the membranes in the stack, the correlation peak intensities were integrated as function of the meridonal angle $\varphi$ (the angle relative to the $q_{z}$ axis) as depicted in Figure $2 b$. The corresponding intensity was fit with a Gaussian distribution centered at 0 , which was then used to calculate the degree of orientation using Hermans orientation function: 
$H=\frac{3<\cos ^{2} \delta>-1}{2}$.

The experimental errors were determined as follows: Errors for peak positions, peak width and peak height are determined as the fit standard errors, corresponding to $95 \%$ confidence bounds, equivalent to two standard deviations, $\sigma$. Errors for calculated parameters, such as peak area, were then calculated by applying the proper error propagation.

Molecular Dynamics Simulation: MD simulations were performed on MacSim, a GPU accelerated computer workstation using GROMACS Version 5.1.4. The computer is equipped with a 40 Core central processing unit (CPU, Intel(R) Xeon(R) CPU E5-2630 v4 @ 2.20GHz), $130 \mathrm{~GB}$ random-access memory (RAM) and three graphic processing units (GPU, $2 \times$ NVIDIA 1080 TDI $+1 \times$ GeForce GT 730). Seven membrane models were designed using the CHARMM-GUI membranebuilder (http://charmm-gui.org// ${ }^{[50,51]}$ and the Martini forcefield 2.2. ${ }^{[51]}$ The systems correspond to a pure red blood cell membrane and membranes containing 10\%, 50\%, and 80\% DMPC respectively. Each system represents a membrane patch of $30 \times 30 \mathrm{~nm}$ with 1500 lipid molecules on each leaflet and 37 water molecules per lipid representing a fully hydrated state of the membrane.

The lipid composition of the membrane patch was chosen according to the widely accepted experimental findings by Dodge et al. ${ }^{[32]}$ However, the presented lipodomic analysis is limited to the ratio of lipid classes and tail saturation. Thus further approximations to the overall lipid composition had to be made. We used the same lipid species presented in the work by Ingólfsson and co-workers ${ }^{[27]}$ and adapted the concentrations respectively to match the aforementioned experimental findings. ${ }^{[32]}$ Note that multiple lipid species are represented by the same arrangement of atoms in the coarse-grained Martini force field. Each membrane system was charge-neutralized by the addition of $(\mathrm{NaCl}$ or $\mathrm{KCl})$ counter-ions. The model is available from the authors upon request.

Simulations were equilibrated for 5 ns using an NPT ensemble (constant pressure and temperature), and then run for $5 \mu \mathrm{s}$. Only the final $3 \mu$ s were analyzed, after affirming the membrane had reached equilibrium by determining the area per lipid. All simulations used a $2 \mathrm{fs}$ time step, a short range van der Waal cutoff of $1.1 \mathrm{~nm}$, a potentialshift-verlet coulomb modifier and periodic boundary conditions were applied to all spacial directions. Neighbor lists were updated in intervals of 20 steps. The temperature coupling was controlled by a $v$-rescale thermostat at a constant pressure of 1 bar using ParrinelloRahman semi-isotropic weak coupling ( $\tau=12$ ps; compressibility $\beta=3 \cdot 10^{-4} \mathrm{bar}^{-1}$ ). DMPC density maps were calculated using the gmx densmap function provided by GROMACS. For this purpose, the phosphate group of DMPC was indexed for each leaflet respectively and the density map was averaged over the last microsecond of the simulation.

The pure RBC membrane model contained 55 different lipid species from five different lipid classes. However, the exact composition was adjusted to produce the desired hybrid membrane models. Figure $4 \mathrm{a}$ shows a 3D render of membranes containing 10, 50, and $80 \mathrm{~mol} \%$ DMPC. The lipid tails are represented by cyan and gray bonds for DMPC and RBC lipids, while blue and red spheres highlight the phosphate group of each membrane species respectively. Details about the exact lipid composition of each model can be found in Table S1, Supporting Information. Figure S3, Supporting Information, visualizes the relative concentrations of lipid species in the membrane model. Time-resolved DMPC density were determined by averaging the in-plane DMPC density over $1 \mu$ s between 2 and $5 \mu$ s in steps of 100 ns. The data were visualized using Matlab and were rendered with ffmpeg (Version 2.8.15).

Epi-Fluorescent Microscopy: Hybrid liposomes were visualized on a Nikon Eclipse Ti2-E inverted microscope, equipped with a CFI Plan Fluor $100 \times$ Oil immersion objective with a numerical aperture of 1.30 and a Tu Plan Fluor BD 50x objective with a numerical aperture of 0.8 . The instrument was used in episcopic illumination mode using a X-Cite 120 LED combined with an excitation filter of $540-580 \mathrm{~nm}$ and an emission filter of 600-660 nm. Images were taken with an Andor Zyla $5.5 \mathrm{sCMOS}$ camera with a resolution of $2560 \times 2160$ pixels and a pixel size of $6.5 \mu \mathrm{m}$ and processed by cropping the image to the size of the liposomes shown. For each picture, brightness and contrast has been adjusted using ImageJ (Version 1.52i). Edges were detected in Image) and the contrast was further increased by adding both the original data and the edge detected picture.

Dynamic Light Scattering and Determination of the Zeta-Potential: The size distribution and the Zeta-potential of the prepared liposomes were determined on a Zetasizer Nano ZS from Malvern Panalytical. The instrument utilizes a $4 \mathrm{~mW} \mathrm{He}-\mathrm{Ne}$ laser (Wavelength: $633 \mathrm{~nm}$ ) in combination with a non-invasive backscattering optics to measure a dynamic light scattering (DLS) spectrum allowing the diffusion constant $D$ of the liposomes to be determined. This is related to the particle size via the Stokes-Einstein relation: $D=\frac{K_{B} T}{6 \pi \eta r}$, where $\eta$ is the dynamic viscosity of the solution, $K_{B}$ is the Boltzmann Constant, $T$ is the sample temperature and $r$ is the radius of a presumably spherical particle. The Zeta-potential is determined by Laser Doppler Micro-Electrophoresis. Here, an alternating electric field is applied to the solution and the velocity of the particles is determined via the patented phase analysis light scattering (M3-PALS, patent reference: US7217350). This allows for the determination of the charge dependent mobility of the particles. All measurements were performed at $25^{\circ} \mathrm{C}$ on $1 \mathrm{~mL}$ sample containing $\approx 14 \mathrm{mg} \mathrm{mL}^{-1}$ of membrane material.

UV-Visible Light Spectroscopy: The encapsulation efficiency and the resistance to mechanical stress was determined using UV-visible light spectroscopy using a Nanophotometer from IMGEN. The liposomes were prepared in a $1 \mathrm{mg} \mathrm{mL} \mathrm{m}^{-1}$ solution of fluorescein dextran, as described above. The liposomes were then isolated by centrifuging for $60 \mathrm{~min}$ at $20000 \mathrm{~g}$, and refilled to a total volume of $2 \mathrm{~mL}$. UVvisible spectra were taken before and after the centrifugation process. Before centrifugation, the absorbance contains contributions from free dextran in the solution and from dextran encapsulated in liposomes. After centrifugation and liposome isolation, only encapsulated dextran contributes to the signal.

The efficiency $E$ is calculated by

$E=\frac{\left(I_{\text {enc }}-I_{\text {pure liposomes }}\right)}{I_{\text {enc }+ \text { free }}}$,

where $I_{\text {enc }}$ and $I_{\text {enc }}+$ free are the integrated intensities of the characteristic absorbance peak of fluorescein $(430-520 \mathrm{~nm})$ for the encapsulated and encapsulated+free dextran molecules, respectively. Corresponding absorbance spectra for hybrid liposomes containing 20\% DMPC are shown in Figure S4, Supporting Information.

The resistance to mechanical stress was determined by a lysis essay. Liposomes were prepared according to the previously described protocol using phosphate buffer saline containing $1 \mathrm{mg} \mathrm{mL}$ fluorescein labeled dextran. The solution was then centrifuged for $60 \mathrm{~min}$ at $20000 \mathrm{~g}$. The supernatant was removed and replaced by phophate buffer saline at varying molar concentrations (1-10 mM) increasing the osmotic and mechanical stress. The samples were allowed to rest for $30 \mathrm{~min}$ and afterward centrifuged for additional $60 \mathrm{~min}$ at $20000 \mathrm{~g}$. The degree of lysis was determined by determine the absorbance of the fluorescein peak. A low degree of lysis results in a low concentration of free dextran in the supernatant while a increased lysis is indicated by a leveled dextran concentration and thus a higher absorbance.

Statistical Analysis: All samples were prepared and measured in replicates and checked for consistency. Errors were determined by the respective experimental errors and consequent error propagation. Details are provided at the appropriate places in the manuscript.

\section{Supporting Information}

Supporting Information is available from the Wiley Online Library or from the author. 


\section{Acknowledgements}

This research was funded by the Natural Sciences and Engineering Research Council of Canada (NSERC), the Canada Foundation for Innovation (CFI) and the Ontario Ministry of Economic Development and Innovation. M.C.R. is the recipient of an Early Researcher Award of the Province of Ontario and a University Scholar of McMaster University. C.W. acknowledges support by the DFG FOR 2688, project WA 1336/12. The funders had no role in study design, data collection and analysis, decision to publish, or preparation of the manuscript.

\section{Conflict of Interest}

The authors declare no conflict of interest.

\section{Keywords}

drug delivery, erythrocyte membrane, hybrid erythrocyte membranes, membrane material properties, red blood cells, synthetic lipids

Received: August 14, 2019

Revised: November 26, 2019

Published online: January 7, 2020

[1] A. Samad, Y. Sultana, M. Aqil, Curr. Drug Delivery 2007, 4, 297.

[2] A. Laouini, C. Jaafar-Maalej, I. Limayem-Blouza, S. Sfar, C. Charcosset, H. Fessi, J. Colloid Sci. Biotechnol. 2012, 1, 147.

[3] B. S. Pattni, V. V. Chupin, V. P. Torchilin, Chem. Rev. 2015, 115, 10938.

[4] L. R. C. Pedrosa, O. van Tellingen, T. Soullié, A. L. Seynhaeve, A. M. Eggermont, T. L. ten Hagen, M. Verheij, G. A. Koning, Eur. J. Pharm. Biopharm. 2015, 94, 207.

[5] T. R. Hoare, D. S. Kohane, Polymer 2008, 49, 1993

[6] L. Sercombe, T. Veerati, F. Moheimani, S. Y. Wu, A. K. Sood, S. Hua, Front. Pharmacol. 2015, 6, 286.

[7] G. M. Ihler, R. H. Glew, F. W. Schnure, Proc. Natl. Acad. Sci. 1973 $70,2663$.

[8] V. R. Muzykantov, Expert Opin. Drug Delivery 2010, 7, 403.

[9] M. Magnani, Am. J. Hematol. 2017, 92, 979.

[10] V. Leuzzi, R. Micheli, D. D’Agnano, A. Molinaro, T. Venturi, A. Plebani, A. Soresina, M. Marini, P. F. Leali, I. Quinti, M. C. Pietrogrande, A. Finocchi, E. Fazzi, L. Chessa, M. Magnani, Neurology-Neuroimmunology Neuroinflammation 2015, 2, e98.

[11] S. Zaitsev, D. Spitzer, J.-C. Murciano, B.-S. Ding, S. Tliba, M. A. Kowalska, O. A. Marcos-Contreras, A. Kuo, V. Stepanova, J. P. Atkinson, M. Poncz, D. B. Cines, V. R. Muzykantov, Blood 2010, $115,5241$.

[12] S. Zaitsev, M. A. Kowalska, M. Neyman, R. Carnemolla, S. Tliba, B.-S. Ding, A. Stonestrom, D. Spitzer, J. P. Atkinson, M. Poncz, D. B. Cines, C. T. Esmon, V. R. Muzykantov, Blood 2012, 119, 4779.

[13] W. M. Armstead, K. Ganguly, J. W. Kiessling, X.-H. Chen, D. H. Smith, A. A. Higazi, D. B. Cines, K. Bdeir, S. Zaitsev, V. R. Muzykantov, J. Cereb. Blood Flow Metab. 2009, 29, 1463.

[14] W. M. Armstead, K. Ganguly, J. Kiessling, J. Riley, X.-H. Chen, D. H. Smith, S. C. Stein, A. A. Higazi, D. B. Cines, K. Bdeir, S. Zaitsev, V. R. Muzykantov, J. Neurochem. 2010, 113, 303.

[15] K. M. Lorentz, S. Kontos, G. Diaceri, H. Henry, J. A. Hubbell, Sci. Adv. 2015, 1, e1500112.

[16] D. Pan, O. Vargas-Morales, B. Zern, A. C. Anselmo, V. Gupta, M. Zakrewsky, S. Mitragotri, V. Muzykantov, PLoS One 2016, 11, e0152074.

[17] V. R. Muzykantov, J. C. Murciano, R. P. Taylor, E. N. Atochina, A. Herraez, Anal. Biochem. 1996, 241, 109.
[18] Y. He, R. Li, H. Li, S. Zhang, W. Dai, Q. Wu, L. Jiang, Z. Zheng, S. Shen, X. Chen, Y. Zhu, J. Wang, Z. Pang, ACS nano 2019, 13, 4148.

[19] Q. Jiang, Y. Liu, R. Guo, X. Yao, S. Sung, Z. Pang, W. Yang, Biomaterials 2019, 192, 292.

[20] Y. Liu, X. Wang, B. Ouyang, X. Liu, Y. Du, X. Cai, H. Guo, Z. Pang, W. Yang, S. Shen, J. Mater. Chem. B 2018, 6, 7033.

[21] S. Himbert, R. J. Alsop, M. Rose, L. Hertz, A. Dhaliwal, J. M. MoranMirabal, C. P. Verschoor, D. M. E. Bowdish, L. Kaestner, C. Wagner, M. C. Rheinstädter, Sci. Rep. 2017, 7, 39661.

[22] R. J. Alsop, R. M. Schober, M. C. Rheinstädter, Soft Matter 2016, 12, 6737.

[23] M. C. Smith, R. M. Crist, J. D. Clogston, S. E. McNeil, Anal. Bioanal. Chem. 2017, 409, 5779.

[24] M. W. Rampling, Biochem. Pharmacol. 1976, 25, 751.

[25] D. Flormann, K. Schirra, T. Podgorski, C. Wagner, Rheol. Acta 2016, $55,477$.

[26] D. Flormann, O. Aouane, L. Kaestner, C. Ruloff, C. Misbah, T. Podgorski, C. Wagner, Sci. Rep. 2017, 7, 7928.

[27] H. I. Ingólfsson, M. N. Melo, F. J. Van Eerden, C. Arnarez, C. A. Lopez, T. A. Wassenaar, X. Periole, A. H. De Vries, D. P. Tieleman, S. J. Marrink, J. Am. Chem. Soc. 2014, 136, 14554.

[28] D. Lingwood, K. Simons, Science 2010, 327, 46.

[29] M. C. Rheinstädter, O. G. Mouritsen, Curr. Opin. Colloid Interface Sci. 2013, 18, 440.

[30] C. L. Armstrong, D. Marquardt, H. Dies, N. Kučerka, Z. Yamani, T. A. Harroun, J. Katsaras, A.-C. Shi, M. C. Rheinstädter, PLOS ONE 2013, 8, e66162.

[31] L. Toppozini, S. Meinhardt, C. L. Armstrong, Z. Yamani, N. Kučerka, F. Schmid, M. C. Rheinstädter, Phys. Rev. Lett. 2014, 113, 228101.

[32] J. T. Dodge, G. B. Phillips, J. Lipid Res. 1967, 8, 667.

[33] V. L. Lew, A. Hockaday, C. J. Freeman, R. M. Bookchin, J. Cell Biol. 1988, 106, 1893.

[34] S. Bhattacharjee, J. Controlled Release 2016, 235, 337.

[35] M. Ribeiro, M. Domingues, J. Freire, N. Santos, M. Castanho, Front. Cell. Neurosci. 2012, 6, 44.

[36] T. Nii, F. Ishii, Int. J. Pharm. 2005, 298, 198.

[37] A. Zaltzman, C. Van den Berg, V. Muzykantov, B. Morgan, Biochem. J. 1995, 307, 651.

[38] V. R. Muzykantov, M. D. Smirnov, A. L. Klibanov, FEBS Lett. 1993, 318, 108.

[39] V. R. Muzykantov, M. D. Smirnov, G. P. Samokhin, Biochimica et Biophysica Acta (BBA)-Biomembranes 1992, 1107, 119.

[40] V. R. Muzykantov, M. D. Smirnov, A. L. Klibanov, J. Immunol. Methods 1993, 158, 183.

[41] C. H. Villa, D. C. Pan, I. H. Johnston, C. F. Greineder, L. R. Walsh, E. D. Hood, D. B. Cines, M. Poncz, D. L. Siegel, V. R. Muzykantov, Blood Adv. 2018, 2, 165.

[42] S. L. Veatch, S. L. Keller, Biochim. et Biophys. Acta (BBA)-Mol. Cell Res. 2005, 1746, 172.

[43] J. Juhasz, F. J. Sharom, J. H. Davis, Biochim. et Biophys. Acta (BBA)Biomemb. 2009, 1788, 2541.

[44] J. Juhasz, J. H. Davis, F. J. Sharom, Biochem. J. 2010, 430, 415.

[45] J. Juhasz, F. J. Sharom, J. H. Davis, Biochim. et Biophys. Acta (BBA)Biomemb. 2009, 1788, 2541.

[46] M. J. Skaug, M. L. Longo, R. Faller, J. Phys. Chem. B 2011, 115, 8500.

[47] N. F. Morales-Penningston, J. Wu, E. R. Farkas, S. L. Goh, T. M. Konyakhina, J. Y. Zheng, W. W. Webb, G. W. Feigenson, Biochimica et Biophysica Acta (BBA)-Biomembranes 2010, 1798, 1324.

[48] A. Khondker, D. J. Malenfant, A. K. Dhaliwal, M. C. Rheinstädter, ACS Infect. Dis. 2018, 4, 926.

[49] J. F. Nagle, M. C. Wiener, Biophys. J. 1989, 55, 309.

[50] S. Jo, T. Kim, V. G. Iyer, W. Im, J. Comput. Chem. 2008, 29, 1859.

[51] Y. Qi, H. I. Ingólfsson, X. Cheng, J. Lee, S. J. Marrink, W. Im, J. Chem. Theory Comput. 2015, 11, 4486. 